\title{
Editorial-The 3rd International Workshop on Modeling the Ocean (IWMO 2011)
}

\author{
Lie-Yauw Oey • Tal Ezer • Bo Qiu • Jarle Berntsen • Ruoying He
}

Received: 7 January 2013 / Accepted: 8 January 2013 /Published online: 22 January 2013

(C) Springer-Verlag Berlin Heidelberg 2013

The 3rd International Workshop on Modeling the Ocean (IWMO; http://service.fio.org.cn/iwmo2011/iwmo2011.htm) was held on June 6-9, 2011 in the beautiful seaport city of Qingdao, hosted by the First Institute of Oceanography, China. The year was marred by the tragic March 11 Tōhoku earthquake a few months before the workshop. This special issue is dedicated to the Japanese people, who despite the enormity of their sufferings, showed immense resilience and fortitude.

Approximately 100 researchers worldwide participated in the Workshop. There were over 80 oral presentations and posters covering, as in past workshops, a broad range of topics on modeling, analysis and observation from smallscale turbulence, waves and air-sea-ice physics to regional, basin and climate-scale processes, and from sediment transport to coupled physical-biogeochemical dynamics. A record of approximately 20 graduate students and postdoctoral students participated in the Outstanding Young Scientist

Responsible Editor: Jörg-Olaf Wolff

L.-Y. Oey $(\bowtie)$

Atmospheric \& Oceanic Science Program, Princeton University,

Princeton, NJ 08544, USA

e-mail: lyo@princeton.edu

T. Ezer

Department of Ocean, Earth \& Atmospheric Sciences,

Old Dominion University, Norfolk, VA, USA

B. Qiu

Department of Oceanography, University of Hawaii, Honolulu, HI, USA

J. Berntsen

Department of Mathematics, Bergen University, Bergen, Norway

R. He

Department of Marine Sciences, North Carolina State University, Raleigh, NC, USA
Competition befitting to the spirit and overall goal of the workshop to encourage young scientists to participate.

The collection of eight papers in this special issue was selected from a total of approximately 30 originally submitted after they underwent the usual process of reviews and revisions. There were at least two reviewers for each paper, but a few of the submissions had three or four reviewers. Some of the reviews were from the workshop attendees, but many were solicited from the scientific community at large.

The subjects of papers in this special issue include four on data assimilations-hindcast and/or test forecast (i.e., using past data but without data assimilation; Isobe et al. 2012; Miyazawa et al. 2012; Yin et al. 2012; Yao et al. 2012), one on process sensitivity (Xing et al. 2012), one on ice floe observation with modeling (Shu et al. 2012), and two on processes with observational and modeling analyses (Ezer et al. 2012; Wada et al. 2012).

Isobe and co-authors (Kako, Guo, and Takeoka) nest a high-resolution finite-volume model (FVCOM; $1 \mathrm{~km}$ near the coast) within the Japan Coastal Ocean Predictability Experiment's (JCOPE2) $1 / 12^{\circ} \times 1 / 12^{\circ}$ reanalysis of the Kuroshio. They conducted test-forecasts of warm intrusion events into the Bungo Channel (132 E and $33 \mathrm{~N}$ ) south of Japan as Kuroshio's frontal waves meander close to the shelfbreak - a challenging feat on account of the sporadic and highly chaotic nature of the processes involved. The ensemble breeding method is used. The experiments successfully predict the occurrences of three observed intrusion events in 2010. The authors suggested that improved lateral boundary conditions from JCOPE2 assimilated with Argo data may further improve the results by eliminating false events produced by the model's (imperfect) physics.

Miyazawa and co-authors (Miyama, Varlamov, Guo, and Waseda) conducted hindcasts of the Kuroshio and coastal circulation south of Japan from January-March of 2010 focusing in the Kii Channel (135 E and $33.6 \mathrm{~N}$ ) where a 
strong sea surface temperature (SST) front is often observed and showed that the front can be realistically simulated. The authors implemented, the first time it seems, the localized ensemble transformation Kalman filter (LETKF) a data assimilation algorithm popular in meteorology [see references in the authors' paper, in particular Hunt et al. 2007] to the mpiPOM (mpi-version of the Princeton Ocean Model) developed by Dr. Jordi (see usage and download information of mpiPOM in Oey et al. (2013)). Ensemble Kalman filtering consists of: (1) choosing an ensemble of, say $k(=20$ are used) initial conditions to calculate error covariance $P,(2)$ propagating each member using the mpiPOM, and (3) computing a new $P$ based on the resulting ensemble at the new time (=+2 days, are used). However, since $k$ is $<<\mathrm{m}$, the dimension of the state vector (=number of prognostic variables $\times$ total number of grid points), the resulting $P$ quantifies uncertainty only in the tiny $k$ subspace and can potentially miss error growths (due to, e.g., baroclinic instability) in other directions of the $\mathrm{m}$ state space. The authors therefore use "localizations" to account for the system's instability driven by local, regional dynamics which can be better represented by the smaller $k$ member ensemble, and the combination of which over the entire model domain can then explore a much higher-dimensional space. This turns out to be crucial for the Kuroshio-Kii Channel SST interaction process due to the spatiotemporally highly chaotic nature of the problem.

A similar ensemble Kalman filtering algorithm is presented by Yin and co-authors (Qiao, Yang, Xia, and Chen). The authors conducted data-assimilative hindcasts of a POM-based western North Pacific $(0-50 \mathrm{~N}$ and 100 $150 \mathrm{E})$ model during 2005 using the ensemble adjustment Kalman filter (EAKF; see references in the authors' paper, in particular, Anderson 2001). This work follows the authors' previous successful attempt at using EAKF for a global ocean model (Yin et al. 2011). The authors assimilated Argo temperature and salinity profiles. They show that compared to runs without data assimilation, the EAKF yields improved variability both for the surface eddy as well as the subsurface temperature and salinity fields when these are checked against independent observational data.

A tidal assimilative modeling is presented by Yao and coauthors (He, Bao, $\mathrm{Wu}$, and Song) who use ROMS at $1 / 30^{\circ} \times$ $1 / 30^{\circ}$ horizontal resolution to calculate $M_{2}$ tide in the Bohai and Yellow Seas (34-42 N, 117-127.4 E). Given coastal tide gauge data, the ROMS' open-boundary elevation and velocities are then adjusted by minimizing (in a least square sense) a cost function involving the error misfit at those coastal stations. The novelty here is that the boundary correction is calculated in an iterative manner using an algorithm based on the linear model TRUXTON which is harmonic in time and which uses finite element discretization in space. An improved $M_{2}$ tidal solution is found.
Xing, Davies, and Jones conducted extensive experiments and analyses using FVCOM to study the temporal and spatial distributions of maximum bottom stress in the eastern Irish Sea as a function of wind and tide-induced currents, as well as also surface source of turbulence due to wave breaking. Wind from different directions with extreme wind stress magnitude of $1 \mathrm{~Pa}$ was tested to model storm surges in conjunction with simulated $M_{2}$ tide specified along the open boundary. The sea water is of constant density but the model solution is three-dimensional. Because of tide-surge interaction, the simulated sea level and currents are modified due to altered bottom stresses as well as wetting and drying in shallow regions near the shore. The maximum bottom stress distributions show complex patterns which are also different for experiments with and/or without the surge. Moreover, while surface source of turbulence due to wave breaking has little or no effects on the maximum bottom stress in deeper water, it significantly changes the bottom stress near the shore where depths $<5 \mathrm{~m}$. Such alterations in the maximum bottom stress can be expected to influence nearshore sediment dynamics and distribution.

Shu, Ma, and Qiao reported observation of an ice floe drift in the Arctic Ocean from 8 to 19 August 2010 by the Chinese Arctic Research Expedition ship. Inertial oscillation with period close to $12 \mathrm{~h}$ is observed as can be expected. The surprising finding is that the ice drifts in the direction to the right of the wind with an averaged turning angle $\approx 40^{\circ}$ which is substantially larger than was previously reported (5-23 $3^{\circ}$ in various seasons) by other investigators. Ice floe models are used to simulate the ice drift, showing inertial oscillation but with much reduced amplitude and no mention of the model turning angle which presumably was also less than the observed. A sea ice-ocean coupled global model is also used which yields similar results. The authors offer neither explanation nor any suggestion as to the source of the large discrepancy between the turning angle found by previous researchers and from their observation.

Ezer and co-authors (Heyman, Houser, and Kjerfve) used a high-resolution (50 m horizontal grid size) POM model to study flow topography interactions near Caribbean coral reefs. The study has implications for small-scale biological-physical processes such as the dispersion of eggs from spawning aggregation cites at Caribbean coral reefs. The case study involves simulations of a very unusual "perfect storm" event recorded in October 2009, where combinations of a local storm, a Caribbean eddy and high tides generated extremely high flows near the tip of a reef and coastal water level gradients as large as $\sim 0.5 \mathrm{~m}$ per $1 \mathrm{~km}$ distance. The model results and a dynamic balance analysis reveal that on those small scales, nonlinear processes play a major role in flow-topography interactions and can create unusually large variations in coastal currents and water levels; these 
processes are fundamentally different than classic winddriven storm surges.

Wada, Waseda, and Nanjo analyze tides in Tsugaru Strait which connects Japan Sea and the North Pacific Ocean. Because of different tidal phases at the two ends, the strait's tidal elevation is semidiurnal $\left(M_{2}\right)$, while currents are predominantly diurnal $\left(K_{1}\right)$. On the other hand, currents measurement off the tip of a peninsula at a narrow portion of the strait indicates the opposite: the diurnal current amplitude is weaker than semidiurnal current amplitude. To study the phenomenon, the authors use POM in two-dimensional (depth average) mode at a resolution $=500 \mathrm{~m}$, force it with tides at the two ends, and also with a steady mean current representing the Tsugaru warm current (TWC) transport of approximately $1.5 \mathrm{~Sv}$ from west to east through the strait. The simulation shows the observed amplitude reversal $\left(M_{2}\right.$ current stronger than $\left.K_{1}\right)$ over a localized region around the peninsula, including also the measurement location. An explanation is given based on the nonzero vorticity flux produced by tidal current separating at the headland, which forms lee-side eddies, and the nonlinear coupling of the resulting residual vorticity with the TWC. The editors wish to congratulate the authors for this solid piece of research, and offer the following complementary interpretations which may further strengthen the authors' explanations. In headland separated flow problems, the source of vorticity is at the boundary, either by the no-slip condition (Tee 1976), bottom slope torque (Signell and Geyer 1991), or corner (Oey 1996). Because of TWC, it is readily seen that by referencing to a frame moving at a constant mean eastward velocity $u_{o}$, the boundary source is larger during the eastward than the westward cycle of the tide, leaving a residual negative vorticity $\left(\zeta_{\text {Res }}\right)$ near the headland. From the authors' last equation on $\mathrm{p}$. 939 (here, $T=$ tide, o=TWC): $-\nabla \cdot\left\langle\mathbf{u}_{\mathrm{T}} \zeta_{\mathrm{T}}\right\rangle \approx \nabla \cdot\left(\mathbf{u}_{\mathrm{o}} \zeta_{\text {Res }}\right)$, which gives $\partial\left\langle\mathrm{u}_{\mathrm{T}}{ }^{2}\right\rangle / \partial \mathrm{y} \approx 2 \mathrm{u}_{\mathrm{o}} \zeta_{\text {Res }}$ (plus an arbitrary divergence-free field) after approximation retaining only the zonal velocity. This shows that, since $\int \zeta_{\text {Res }}$ dy $<0$ near the headland, the tidal amplitude $\left\langle\mathrm{u}_{\mathrm{T}}{ }^{2}\right\rangle \sim 2 \mathrm{u}_{0} \zeta_{\text {Res }}$ dy, i.e., it is a decreasing function of TWC, as seen in Fig. 9. As pointed out by the authors, since the strait's current is diurnal, the effect is largest on the diurnal current. However, the above holds also for the $M_{2}$ current—see Fig. 9.

We wish to thank our host Dr. Fangli Qiao, Deputy Director of the First Institute of Oceanography, and FIO scientists and staff for their warm hospitality and help during the workshop. We also thank the Chief Editor Dr. JörgOlaf Wolff of Ocean Dynamics for help and encouragements throughout the process of preparing this Special Issue. Last but not least, we thank all the reviewers for their help in ensuring that the submitted manuscripts are held to a high standard.

\section{References}

Ezer T, Heyman WD, Houser C, Kjerfve B (2012) Extreme flows and unusual water levels near a Caribbean coral reef: was this a case of a "perfect storm"? Ocean Dyn 62:1043-1057. doi:10.1007/ s10236-012-0545-5

Isobe A, Kako S, Guo X, Takeoka H (2012) Ensemble numerical forecasts of the sporadic Kuroshio water intrusion (kyucho) into shelf and coastal waters. Ocean Dyn 62:633-644. doi:10.1007/ s10236-011-0519-Z

Miyazawa Y, Miyama T, Varlamov SM, Guo X, Waseda T (2012) Open and coastal seas interactions south of Japan represented by an ensemble Kalman Filter. Ocean Dyn 62:645-659. doi:10.1007/ s10236-011-0516-2

Oey L-Y (1996) Flow around a coastal bend. J Geophys Res 101:16,667-16,682

Oey L-Y, Chang Y-L, Lin Y-C, Chang M-C, Xu F-H, Lu H-F (2013) ATOP - the Advanced Taiwan Ocean Prediction System based on the mpiPOM Part 1: model descriptions, analyses and results. Terr Atmos Ocean Sci 24. doi:10.3319/TAO.2012.09.12.01(Oc), Available from: http://mpipom.ihs.ncu.edu.tw/PUBLICATION/ Oey-etal-ATOP-TAO.pdf

Shu Q, Ma H, Qiao F (2012) Observation and simulation of a floe drift near the North Pole. Ocean Dyn 62:1195-1200. doi:10.1007/ s10236-012-0554-4

Signell RP, Geyer WR (1991) Transient eddy formation around headlands. J Geophys Res 96:2,561-2,575

Tee K (1976) Tide-induced residual current, a 2-D nonlinear numerical tidal model. J Mar Res 34:603-628

Wada R, Waseda T, Nanjo H (2012) Nonlinear interaction of the Tsugaru Warm Current and tide in the Tsugaru Strait. Ocean Dyn 62:923-941. doi:10.1007/s10236-012-0535-7

Xing J, Davies AM, Jones JE (2012) Influence of sea surface wind wave turbulence upon wind-induced circulation, tide-surge interaction and bed stress. Ocean Dyn 62:1017-1042. doi:10.1007/ s10236-012-0542-8

Yao Z, He R, Bao X, Wu D, Song J (2012) M2 tidal dynamics in Bohai and Yellow Seas: a hybrid data assimilative modeling study. Ocean Dyn 62:753-769. doi:10.1007/s10236-011-0517-1

Yin X, Qiao F, Yang Y, Xia C, Chen X (2012) Argo data assimilation in ocean general circulation model of Northwest Pacific Ocean. Ocean Dyn 62:1059-1071. doi:10.1007/s10236-012-0549-1 